\title{
Research on the Current Situation and Development of Chaozhou Opera in Thailand
}

\author{
Lei Miao \\ Guangdong University of Foreign Studies, Guangzhou, China
}

Keywords: Chaozhou Opera, Thailand

Abstract: Thailand is an important country on the maritime Silk Road. Chaozhou Opera has a long history in Thailand. At present, it plays an important role in the transmission of national spirit, but it also faces new opportunities and challenges. Therefore, this paper analyzes the current situation of Chaozhou Opera in Thailand from the perspective of cultural communication, and puts forward corresponding solutions and innovative paths for the shortcomings in the communication process.

\section{Introduction}

Chaozhou Opera, one of the many variants of Chinese opera, has a time-honored history that is much longer than Beijing Opera and Yue Opera. Chaozhou Opera, also known as Chao opera, is one of the ten major operas in China, which is among the first batch in the National Intangible Cultural Heritage Protection List. It is popular in the Chaoshan, Guangdong province and is an ancient local opera sung in Chaozhou dialect. In 2015, The History of Chaozhou Opera, co-authored by $\mathrm{Wu}$ Guoqin and Lin Chunjun, revealed that Chaozhou Opera was the result of long-time exchanges of Nanxi Opera and the local art of Chaozhou [1], confirming that the traditional Chaozhou Opera was the combination of the local culture and vocal tunes of East Guangdong and South Fujian, rather than originating from ancient Chinese music of Guanxitong (an ancient Chaozhou witchcraft of inviting the patron saint of Chinese regional opera)or Zhengzi Opera. From the above, the origin of Chaozhou Opera has been clearly stated. Chaozhou Opera has now become an important local opera in China and is growing mature through innovative practices. Due to the rise of ancient Maritime Silk Road, Chaozhou Opera has become one of the most influential local operas in China. It has gone abroad together with Chaozhou people, serving as an emotional support of many overseas Chinese, and has become one of the most influential Chinese operas overseas. Chaozhou Opera is very popular in Southeast Asia, especially in Thailand, which is known as its second hometown. Chaozhou Opera was spread to Thailand hundreds of years ago with Chaoshanese people, serving as a cultural bond connecting Chaoshan and other countries in the world.

\section{The Spreading History and Significance of Chaozhou Opera in Thailand}

\subsection{The Spreading History of Chaozhou Opera in Thailand}

As early as the middle of the 17th century, Chaozhou opera was introduced into Thailand with immigrants. Before the 1920s, Thailand's Chaozhou Opera was basically a traditional Chinese opera. From the 1920s to the 1940s, Chaozhou Opera set off a climax in Thailand. [2] Great pressure also came from Thai policies. In 1947, Chinese schools at all levels were required to be 
registered by Thailand's Ministry of Education in accordance with the Regulations of Private Schools, so new Chinese schools and existing Chinese middle schools were not allowed. In 1948, a strict repression of Chinese education was initiated, and 53 people from the Thai Chinese Education Association and Nanyang Middle School were arrested by the military government on June 15th of the same year (commonly known as the Anti-Chinese Repression). Consequently, more Chinese schools were closed and there were only 230 Chinese schools (4-year lower primary schools) in the whole Thailand in 1951 [3]. A series of restrictions on Chinese education by Thai government resulted in the decreasing number of Chaozhou-dialect speakers in the younger generation of Thai Chinese and local people, as well as those fond of Chaozhou Opera. As a consequence, the development of Chaozhou Opera fell into a serious depression in Thailand. Later in the 1950s and 1960s, performances of Chinese Chaozhou Opera troupes were almost canceled in Thailand due to political reasons of the two countries. On July 1st, 1975, since Thailand and China established formal diplomatic relations, the two countries have developed an increasingly mature relationship, laying a solid foundation for enhancing economic and trade exchanges, cultural interconnectivity and mutual understanding between the two peoples.

\subsection{The Significance of Chaozhou Opera in Thailand}

With the development of global economy and political multipolarization, the world is moving towards cultural diversity. Cultural exchange has gradually become an important issue for all countries. The initiative of "One Belt, One Road" is the best way to create a cooperative, peaceful and harmonious external cooperation environment for China at present. The initiative of "One Belt, One Road" clearly pointed out that we should strengthen exchanges and cooperation in regional culture on the "maritime Silk Road", enhance exchanges, strengthen cooperation in cultural fields and enhance centripetal force of cooperation. The current economic development of China has exerted significant influence on the economy of ASEAN countries and the world at large. Moreover, China is a major trade partner of Thailand and plays an important role in Thailand's tourism development. The harmonious relations between China and Thailand promote the development of domestic undertakings of the two countries. Chaozhou Opera has a profound cultural heritage and unique artistry. Through the communication and exchange of Chaozhou Opera in Thailand, Thai people can deeply understand the core values of Chinese traditional culture, which is conducive to further promoting the international recognition of Chaozhou Opera culture and enhancing the soft power and international status of Chinese culture. Since cultural communication is the key for people's communication, Chaozhou Opera should follow the trend of times and play an active role in promoting mutual communication and trust between the people of China and Thailand. In addition, the spread of Chaozhou Opera in Thailand is conducive to the recognition and closeness of Thai overseas Chinese and other overseas people to China, further enhancing national self-esteem and self-confidence, and actually playing a role of cultural communication messenger.

\section{The Challenges Faced by the Spread of Chaozhou Opera in Thailand}

\subsection{Lack of Diversification of Communication Methods}

Most of Chaoshanese people adhere to the folk customs. Even if they move to Thailand, they will still keep their native customs. On the important days, they will continue to worship their ancestors and gods in the temple, and at the important worship activities, they will hire a drama team to perform to show their respect. Such traditional performance methods have been unable to meet the needs of the development of reality. Communication effect usually depends on the content of communication, and the effect of communication content through different communication methods 
is not the same. Due to various reasons, the current communication mode of Chaozhou Opera in Thailand is still lack of diversity. The relevant cultural institutions of China and Thailand should carry out various forms of exchange, such as Chaozhou Opera lectures, course teaching, drama performance, etc. On the one hand, they can improve the artistic quality of local Chaozhou Opera workers in Thailand, on the other hand, they can exchange academic information of Chaozhou Opera culture with each other, so as to promote the development of Chaozhou Opera in Thailand.

\subsection{Limited Audience and Shrinking Market}

The innovation and development of science and technology have had a tremendous impact on society and people's lifestyle. With the rapid development of mobile network, the world has ushered in a new era of we-media, and the way people consume and entertain has also changed. Chaozhou Opera, one of the treasures of traditional Chinese culture, has also been greatly impacted. Its audience at home and abroad is decreasing day by day, and young audience is even fewer. In this sense, Chaozhou Opera is almost in an embarrassing situation. Since the spread of Chaozhou Opera can be seen as a process of symbolic communication, its spreading effect is closely related to the media and the acceptance mode of audience. In Thailand, with the passing of older generation of Chaozhou audience, the third and fourth generation of Chaozhou immigrants have a strange sense of Chaozhou Opera Culture. In addition, the spread of Chaozhou Opera in Thailand is also faced with some serious practical difficulties, such as language barriers, lack of successors of Chaozhou Opera actors, and the market is gradually shrinking.

\subsection{Poor Feedback Channel and Unsatisfactory Effect}

The five basic elements of communication---subject, contents, audience, channel and effect---are interacted in the communication process. Feedback and evaluation are important links to test the effect of communication. At present, the construction of communication evaluation system matching with the communication of Chaozhou Opera Culture in Thailand is still in a state of serious lag or even absence. It is an important problem to establish a comprehensive evaluation feedback mechanism including audience, professional actors, copyright brokers, overseas troupes, media, etc.

\section{The Innovative Strategy of Chaozhou Opera in Thailand}

\subsection{Innovate the Content and Form of Chaozhou Opera}

From the perspective of cultural communication, in order to impress the Thai audience or readers, works of Chaozhou Opera should not only express the major concerns of human beings, but also show the unique aesthetic taste of the nation. Therefore, it is necessary to intensify efforts to cultivate Chaozhou Opera writers in Thailand. Based on telling "China's stories" well, these writers, who are familiar with the rules of creating Chaozhou Opera and Thai history and culture, have adapted and created Chaozhou Opera works according to Thai folk stories, historical allusions, classical films and TV works that conform to the common values of the two peoples. These cultural works of Chaozhou Opera in the new era are both innovative and well-established. They integrate the classics in ancient and modern times both at home and abroad, and will attract more and more Thai audiences. 


\subsection{Integrate Resources and Promote Communication in an All-Round Way}

To further promote Chaozhou Opera in Thailand, it is not enough to rely on government sectors, publishing houses or opera troupes of China. Instead, it is necessary to connect all relevant parties at home and abroad, and establish a multi-dimensional and diversified international spreading channel. It is thought to be an effective way to promote Chaozhou Opera through the Confucius Institutes and Confucius Classrooms in Thailand. The Confucius Institute is a non-profit educational institution advocated by China, and also an important platform to deepen the understanding of Chinese culture and strengthen cultural cooperation and exchanges among people in the world. By 2019, there were 16 Confucius Institutes and 11 Confucius Classrooms in Thailand. The number of Confucius Institutes in Thailand ranked first among Asian countries [4]. The in-depth cooperation between the relevant parties and Confucius Institutes and Confucius Classrooms in Thailand will effectively promote the publication and spread of Chaozhou Opera. At the same time, the domestic "brand marketing" of Chaozhou Opera should also be paid attention in the same time. Chaozhou Opera can be effectively promoted through classical culture appreciation clubs and traditional cultural forums to Thai students, Thai embassies and consulates in China, as well as Thai scholars and journalists in China, so that they can spontaneously help to spread Chaozhou Opera and build a bridge of cultural communication between China and Thailand.

\subsection{Strengthen International Cooperation and Exchange to Achieve Win-Win Cooperation}

China and Thailand should attach importance to and strengthen the cultural exchange of Chaozhou Opera, absorb nutrition from many sides, so as to enhance its vitality. Through official and non-governmental organizations, we will strengthen the close exchanges and interaction between Chaozhou Opera performance groups, professional colleges and full-time teachers in the two countries. Thailand's Chaozhou Opera troupe should pay attention to introducing professional forces from China as the main creators, and also employ professional Chaozhou Opera actors and creators to engage in local teaching and research through a long-term and short-term combination in teaching. The cultural exchanges between China and Thailand not only promote the spread of Chaozhou Opera in Thailand, but also make the development of Thai opera in the international vision system.

\section{Conclusion}

Chaozhou Opera is a famous local opera art in China, which has an important influence not only in China, but also at abroad especially in Southeast Asia. The inheritance and development of Chaozhou Opera in Thailand has made outstanding contributions to its development. China and Thailand should further strengthen their exchanges of Chaozhou Opera art, publicizing and protecting traditional Chaozhou Opera, as well as learning from other arts and getting innovative. Therefore, Chaozhou Opera will make new contributions to promoting friendly exchanges between the two peoples.

\section{REFERENCES}

[1] Wu, G.Q., Lin, C.J. (2015) The History of Chaozhou Opera (First Volume). Guangzhou: Huacheng Press.

[2] Chen Q.X. (2012) Study on Development and Evolution of Chaozhou Opera in Thailand. Chongqing: Chongqing University.

[3] Gu M.Y. (1998) Great Dictionary of Education. Shanghai: Shanghai Education Press.

[4] http://www.hanban.org/confuciousinstitutes/node_10961.htm 